Columbia Law School

Scholarship Archive

2012

\title{
The Measure of a MAC: A Quasi-Experimental Protocol for Tokenizing Force Majeure Clauses in M\&A Agreements
}

\author{
Eric L. Talley \\ Columbia Law School, etalley@law.columbia.edu \\ Drew O'Kane \\ deokane@gmail.com
}

Follow this and additional works at: https://scholarship.law.columbia.edu/faculty_scholarship

Part of the Banking and Finance Law Commons, Business Organizations Law Commons, and the Contracts Commons

\section{Recommended Citation}

Eric L. Talley \& Drew O'Kane, The Measure of a MAC: A Quasi-Experimental Protocol for Tokenizing Force Majeure Clauses in M\&A Agreements, 168 JITE 181 (2012).

Available at: https://scholarship.law.columbia.edu/faculty_scholarship/1699

This Article is brought to you for free and open access by the Faculty Publications at Scholarship Archive. It has been accepted for inclusion in Faculty Scholarship by an authorized administrator of Scholarship Archive. For more information, please contact scholarshiparchive@law.columbia.edu, rwitt@law.columbia.edu. 


\title{
The Measure of a MAC: A Machine-Learning Protocol for Analyzing Force Majeure Clauses in M\&A Agreements
}

by

Eric Talley and Drew O'Kane*

\begin{abstract}
This paper develops a protocol for using a familiar data set on force majeure provisions in corporate acquisitions agreements to tokenize and calibrate a machinelearning algorithm of textual analysis. Our protocol, built on regular expression (RE) and latent semantic analysis (LSA) approaches, serves to replicate, correct, and extend the hand-coded data. Our preliminary results indicate that both approaches perform well, though a hybridized approach improves predictive power further. Monte Carlo simulations suggest that our results are generally robust to out-of-sample predictions. We conclude that similar approaches could be used more broadly in empirical legal scholarship, especially including in business law. (JEL: C63, C81, C88, K00, K12, K22)
\end{abstract}

\section{Introduction}

In recent years, the field of empirical legal studies (ELS) has generated a wealth of academic scholarship that is impressive in both breadth and reach. Though anomalous and rudimentary (at best) within the legal scholarship two decades ago, empirical methods now permeate virtually every area of law, including (but not limited to) administrative law, constitutional law, corporate/securities law, employment law, civil procedure, and jurisprudence.

To be sure, a driving force behind the recent growth and success of ELS is the influx of legal scholars with formal training in empirically sophisticated methodologies, such as economics, psychology, statistics, and sociology. But in addition, empirical methods have also gained traction because of the expansion of publicly available data. As published opinions, regulations, transactional forms, and other

* Eric Talley (corresponding author) and Drew O'Kane are at UC Berkeley (Boalt Hall) School of Law. Thanks to Zev Eigen, Christoph Engel, Christian Kellner, Kevin Quinn, Justin McCrary, Alexander Stremitzer, Matthew Taddy, and seminar participants at UC Berkeley, the University of Texas, Stanford, UC Hastings, the 2011 Conference on Empirical Legal Studies and the 2011 JITE conference on "Testing Contracts" for comments and suggestions. Thanks as well to John Lee for excellent research assistance. All errors are ours.

Journal of Institutional and Theoretical Economics

JITE 168 (2012), 181-201 @ 2012 Mohr Siebeck - ISSN 0932-4569 
legal documents have become increasingly available in digital form in the mid 1990s, numerous scholars interested in empirical methods had - for the first time a significant amount of usable raw material to synthesize, interpret, parse, describe, analyze, and test.

Nevertheless, a significant roadblock continues to hamper the practical feasibility of empirical methods: the fact that empirical legal scholarship tends (by definition) to be "hard," numerical and quantitative, while most original legal sources tend - like the practice of law itself - to be nuanced, textual and qualitative. Consequently, for those interested in pursuing empirical questions in law, it is still the norm to rely on human filters as transcription vehicles, asking students, researchers, or practicing attorneys to read, parse, classify, and summarize quantitative data from original texts. In many ways, this human element is unavoidable (and even desirable), since the practice of law is in many ways the art of navigating between nuanced forms of expression and hard legal outcomes or predictions. Moreover, the process of hand coding allows those inputting data to make nuanced judgments about subtle differences in detail between raw data sources (such as transactional documents from different jurisdictions) and how such sources should be treated (e.g., incorporating whether applicable law is, say, immutable in one jurisdiction and default rules the other).

In many areas of legal practice, this appreciation for nuance can be invaluable. In empirical scholarship, however, exclusive reliance on human coding of legal texts can also impede one's ability to marshal the power of textual legal datasets. Perhaps the most substantial roadblock is cost. Unlike other forms of hand coding, human coding of legal sources generally requires personnel with legal expertise in the topical area of interest. Within most developed legal systems, this generally means enlisting practicing lawyers or advanced law students to do the work. But for either group, opportunity costs and outside prospects can be significant, driving up the costs of collection. Moreover, such datasets not only entail significant startup expenditures, but their marginal maintenance and updating costs remain high as well. In short, hand coding raw legal and regulatory data is an arduous and painstaking process.

A related roadblock concerns internal reliability and consistency of a hand-coded dataset: given the human capital and time requirements of hand coding large-scale, longitudinal projects, such endeavors often involve a revolving door of personnel. The practice of making judgment calls, relative attention to nuance, and the legal backgrounds of contributors vary wildly, both within coders and between them over time. It is often difficult if not impossible to know the nature and direction of resulting biases, rendering corrective measures challenging at best, prohibitive at worst.

During much of the time in which empirical legal scholarship has been developing, computer scientists and natural language theorists have been developing tools (largely - though not exclusively - outside of the legal context) for the large-scale automated analysis of textual data. Pioneering developments in these fields, many of which have emerged in the last fifteen years, have already come to dominate much 
of computational biology, library sciences, and information theory. (Indeed, the core business of companies like Google has and remains in the management, organization, and indexing of vast quantities of qualitative data.) Although natural language processing approaches are now starting to infiltrate legal practice and scholarship (particularly in the areas of e-discovery and analysis of judicial opinions), they have tended to do so in a way that is independent of and parallel to traditional forms of database development within legal scholarship. In most private/transactional law contexts, moreover, these approaches are virtually nonexistent.

In this paper, we propose a method for using expensive, lawyer-coded databases to calibrate (or "tokenize") a machine-learning protocol for replicating, correcting, and significantly expanding those databases. Effectively, our approach treats existing hand-coded data as a type of calibration instrument, embodying assessments, nuanced judgments, and resulting regularities that can provide the basis automated probabilistic coding protocol, with potential improvements in reliability, cost, scalability, and speed over conventional methods. Our specific focus is on mergers and acquisitions (M\&A) agreements executed between 2007 and 2008, and in particular the use of (so-called) "Material Adverse Change" or "Material Adverse Event" (MAC/MAE) provisions in such agreements. MAC/MAEs are a central workhorse provision for allocating risk and uncertainty that can emerge between the execution of a corporate acquisition and its closing - a period that can often take many months (and sometimes years). Such provisions typically condition one party's (almost always the buyer's) obligation to close an M\&A transaction on the absence of any occurrence, condition, change, event, or effect that materially and adversely affects some enumerated dimension of the deal's value. When triggered, a MAC/MAE provision effectively gives the advantaged party the right to "walk away" from an executed deal (or at least to threaten to do so as a backdrop to renegotiation). Consequently, MAC/MAE provisions tend to be a central focus of negotiating parties during an acquisition - they are truly "dickered" as part of the deal. (See, e.g., Talley, 2009; Gilson and Schwartz, 2005; Macias, 2009.)

To provide a training data set for our classification model, we make use of a well known attorney-coded database that tracks the presence/absence of twenty different MAC/MAE sub-provisions in announced agreements executed between 2007 and 2008, and previously analyzed in Talley (2009). Combining this data with the raw text from each deal's MAC/MAE provision, we develop two predictive machinelearning protocols - both built largely on Python computer code - for diagnosing the presence of each sub-provision. The first protocol is based on a "Regular Expression" (RE) algorithm - a Boolean dictionary that summarizes syntactical patterns that are characteristic of each type of contractual sub-provision. The second protocol utilizes "Latent Semantic Analysis" (LSA) techniques for analyzing the raw content of each provision by generating a frequency table of its terms (a metaphorical "bag of words" inventorying the terms used in each MAC/MAE provision). Each of the RE and LSA approaches is capable of generating relatively accurate calibrations that perform reasonably well in replicating the hand-coded data (both achieved overall within-sample classification accuracies of approximately $80 \%$ ). 
However, we further demonstrate that when used in combination with one another, RE and LSA methodologies perform even better (producing within-sample classification accuracies in the $85 \%$ range). We also employ Monte Carlo methods to simulate the out-of-sample predictive power of each methodology (and both in combination), and our results remain qualitatively similar to (though, not surprisingly, a bit weaker and noisier than) the full-sample calibration. Overall, on the basis of this exercise, we conclude that our protocol provides a promising proof of concept for replicating, correcting, and significantly expanding the depth and breadth of existing hand-coded legal datasets, at a significant marginal cost savings.

Before proceeding, we pause to highlight an important caveat regarding the relationship of our approach to traditional approaches in empirical legal scholarship. It is perhaps enticing to think that machine-learning classifiers (such as those developed here) represent a substitute for the arduous, laborious hand-coding protocols that generate most existing empirical legal datasets. This temptation is, however, nearly as incorrect as it is tempting. Indeed, virtually any text classifier (including ours) requires a preexisting "training" data set against which to calibrate its predictive model. Without such training data, our approach would have little (if anything) to commend it. In this sense, then, our approach represents an important complement (rather than substitute) for hand-coded data. We conjecture that approaches similar to ours are likely to be particularly promising tool for those currently building or maintaining legal scholarship data to improve it further.

Moreover, the general approach championed here can plausibly be carried over to quasi-experimental settings as well. Nothing necessarily requires that training data be drawn from a pre-existing data source - it could also come from (for example) laboratory or field experimental manipulations. For example, we are currently piloting an extension to this study where subjects in a laboratory are asked to evaluate, negotiate, and "price out" a set of specimen MAC/MAE provisions based upon our dataset within a hypothetical deal. This approach - if successful - will allow us not only to calibrate a predictive model of the presence/absence of certain canonical provisions (as done here), but it will permit us to assemble a cardinal monetary measure of the "buyer-friendliness" of each provision based on its constituent parts - one that can possibly also extrapolated outside the experimental sample. We therefore view the current project as a first (but important) step in combining both empirical and experimental data with machine-learning approaches for large quantitative text analysis within law.

Our analysis proceeds as follows. Section 2 describes MAC/MAE provisions in greater detail, and briefly discusses their significance in corporate law and M\&A practice. Section 3 describes our data and general methodology, including the RE and LSA protocols we develop for building our algorithmic models. Section 4 presents our tentative results, for both full-sample calibrations and for simulated out-of-sample predictions using Monte Carlo methods. Section 5 discusses our results in greater detail, considers a number of extensions to our approach, and offers concluding remarks. 


\section{Background: What Is a MAC?}

As noted above, our paper utilizes a pre-existing lawyer-coded database of MAC/ MAE provisions as a training template for a predictive natural language processing protocol. To better motivate our enterprise, this section spends some time describing what, exactly, these provisions are, what purposes they serve, and how they come into existence.

MAC/MAEs are a species of contractual force majeure (or "act of God") provision peculiar to acquisitions and financing transactions. Put simply, the MAC/MAE functions as a type of express condition on one party's (or sometimes both parties') obligation to complete performance an executed contract. As a matter of legal formality, most MAC/MAEs in M\&A and financing deals are construed to be conditions subsequent: i.e., the occurrence of the enumerated contingency relieves the advantaged party of her pre-existing duty to close. In some situations, however, the MAC/MAE can be construed as a condition precedent, whereby the advantaged party has no duty to perform unless the enumerated contingency obtains. Although this difference is in some ways semantic, it has practical implications - for it effectively determines which party must bear the evidentiary burden of demonstrating that a triggering condition has occurred.

Regardless of how it is construed for evidentiary purposes, the substantive architecture of a typical MAC/MAE provision is perhaps best described as resembling a (metaphorical) piece of Swiss cheese. ${ }^{1}$ One portion of the provision ("affirmative" section) usually appears at the beginning of the provision, and constitutes the cheesy bit, enumerating often broad categories of contingencies where a material change in circumstance relieves the buyer of her obligation to close. Another portion of the MAC/MAE provision (the "exclusion" or "carve-out" section) invariably follows, and constitutes the metaphorical holes in the cheese. The exclusions typically take the form of a more lengthy and specific list of enumerated contingencies that do not constitute an escape hatch for performance, notwithstanding the broad affirmative provisions.

The MAC/MAE provision in the 2007 acquisition of the Huntsman Corporation (which culminated in the litigated case of Hexion v. Huntsman (2008)) provides an apt example of this architecture. The MAC/MAE from that deal is reproduced in its entirety below, with the affirmative portion underlined, and the carve-outs in italics:

A "Company Material Adverse Effect" means any occurrence, condition, change, event or effect that is materially adverse to the financial condition, business, or results of operations of the Company and its Subsidiaries, taken as a whole; provided, however, that in no event shall any of the following constitute a Company Material Adverse Effect: (A) any occurrence, condition, change, event or effect resulting from or relating to changes in general economic or financial market conditions, except in the event, and only to the extent, that such occurrence, condition, change, event or

1 We give due acknowledgment co-author Talley's 9-year old daughter, Gracie, whose culinary obsession with Mac and Swiss cheese provided the initial inspiration for this metaphor. 
effect has had a disproportionate effect on the Company and its Subsidiaries, taken as a whole, as compared to other Persons engaged in the chemical industry; (B) any occurrence, condition, change, event or effect that affects the chemical industry generally (including changes in commodity prices, general market prices and regulatory changes affecting the chemical industry generally) except in the event, and only to the extent, that such occurrence, condition, change, event or effect has had a disproportionate effect on the Company and its Subsidiaries, taken as a whole, as compared to other Persons engaged in the chemical industry, $(C)$ the outbreak or escalation of hostilities involving the United States, the declaration by the United States of war or the occurrence of any natural disasters and acts of terrorism, except in the event, and only to the extent, of any damage or destruction to or loss of the Company's or its Subsidiaries' physical properties; (D) any occurrence, condition, change, event or effect resulting from or relating to the announcement or pendency of the Transactions (provided, however, that this clause (D) shall not diminish the effect of, and shall be disregarded for purposes of, the representations and warranties relating to required consents, approvals, change in control provisions or similar rights of acceleration, termination, modification or waiver based upon the entering into of this Agreement or consummation of the Merger); (E) any change in GAAP, or in the interpretation thereof, as imposed upon the Company, its Subsidiaries or their respective businesses or any change in law, or in the interpretation thereof; $(F)$ any occurrence, condition, change, event or effect resulting from compliance by the Company and its Subsidiaries with the terms of this Agreement and each other agreement to be executed and delivered in connection herewith and therewith (collectively, the "Transaction Agreements"), actions permitted by this Agreement (or otherwise consented to by Parent) or effectuating the Financing; or $(G)$ any occurrence, condition, change, event or effect resulting from or in connection with any Divestiture Action ...

Even a cursory inspection of this provision yields a few immediate observations. First, while this MAC/MAE provision is of roughly typical length (422 words), it is far from Hemingwayesque, and it contains significant detail. Second, the affirmative provision of the MAC/MAE is brief and drafted in sweeping terms (applying to a shock in circumstances that materially affects the seller's financial condition, business, or results of operations); the exceptions, in contrast are spelled out in significantly more precise, tedious details. Although this MAC/MAE provision was recognized even at the time as having a fairly large number of seller friendly carveouts, ${ }^{2}$ this general pattern persists across all deals studied here.

Within the larger merger agreement, a MAC/MAE might typically be found in one of many different locations. In some cases it appears as a stand-alone provision, delineated separately from other terms, and specifically granting the favored party a contingent right to walk away. In other deals, the MAC/MAE is incorporated into the representations and warranties, explicitly tied to a "bring down" provision that effectively scuttles the merger when the MAC/MAE is triggered. In yet other cases, the MAC/MAE appears as an embedded component of the closing conditions to

2 The New York Times DealBook, "Huntsman-Hexion: A Deal Agreement to Applaud," available for download at http://dealbook.blogs.nytimes.com/2008/01/11/ huntsman-hexion-a-deal-agreement-to-applaud/ (Jan. 11, 2008, 16:34 EST). 
a deal. In yet other deals, the MAC/MAE may be found spread across two or more of these sections of an acquisition agreement.

As the Huntsman excerpt implicitly suggests, negotiating teams often spend a significant amount of time dickering the precise terms of MAC/MAE provisions. The provisions have accordingly garnered a great deal of attention from both academics and legal professionals. Theories abound as to what purpose the MAC/MAE plays in an acquisitions agreement that is unique from other risk allocation devices (such as contingent prices, earn outs, termination fees, indemnities and guarantees, and the like). One prominent theory (Gilson and Schwartz, 2005) posits that MAC/MAE provisions optimally impose risk on the target's management, which would otherwise have poor incentives to maintain firm value in the interim period between execution and closing. Others (Talley, 2009) have argued that MAC/MAE provisions are uniquely well suited to allocate ambiguity about the mutual gains from the deal (as distinct from risk). Still others (Choi and Triantis, 2010) have argued that MAC/MAE provisions are calibrated to facilitate deal restructuring with minimal transaction costs. It is likely that each of these explanations plays a role in explaining the purposes behind MAC/MAE provisions. For the purposes of this paper, we need not adjudicate among these competing theories. We simply note that each of them is, in principle, testable with sufficient empirical data. And the purpose of this project is to suggest a way to enhance and improve that data.

\section{Data and Methodology}

The significance that MAC/MAE provisions have for practicing lawyers is reflected in the considerable interest within private and academic circles. Notable among these efforts is a longstanding database built by the New York law firm of Nixon Peabody LLP, a firm with a substantial mergers and acquisitions practice. In part as a client development service, Nixon Peabody produces a survey of MAC provisions, publishing summary statistics in an annual publication used widely in the industry (e.g., Nixon Peabody LLP, 2008). The firm has been producing its annual survey for over a decade (Nixon Peabody LLP, 2008, p. 2), and since 2005 its methodology has become sufficiently consistent to be somewhat usable in time series analysis. We obtained access to this database, and sampled 123 acquisitions agreements coded in the 2007-08, corresponding with those involving public targets and for which we could obtain the full merger agreements from publicly available sources (usually the SEC's Edgar database). The plurality of the deals $(45.5 \%)$ involve stock mergers, followed by cash mergers $(30 \%)$, negotiated tender offers $(11 \%)$, stock purchases $(9 \%)$, and asset sales $(4 \%)$. This appears representative of deals occurring during the same time span (Talley, 2009). In what follows, we will periodically refer to the Nixon Peabody lawyer-coded data set as our "training" database.

All coding for the Nixon Peabody data was done by practicing members of the New York bar, usually (but not wholly) comprised of early- to mid-level 
associates. ${ }^{3}$ For each MAC/MAE in a deal $i$, the coding attorney was required to identify a vector $\left\{y_{i 1}, y_{i 2}, \ldots, y_{i k}\right\}$ of binary "attributes," effectively indicating the presence/absence of specifically enumerated sub-provisions that might be included in the MAC/MAE. Consistent with the description above, attorneys were asked to code both "affirmative" MAC/MAE clauses (e.g., terms that deal with the target's financial condition, the seller's ability to close the deal, the target company's prospects, etc.), and exceptions or "carve-outs" (such as changes to the economy in general, changes to securities markets, interest rates, GAAP, etc.). As a general matter, the presence of affirmative MAC/MAEs tends to expand the breadth (and the buyer-friendliness) of the provision, while carve-outs tend to contract that breadth. For our analysis, we focused on 20 coded provisions, selected (largely) at random from the entire set of 44 MAC/MAE provisions coded in the Nixon Peabody dataset. An inventory, brief descriptions and sample frequencies of these provisions are contained in Table 1 below.

As Table 1 illustrates, there are seven affirmative MAC/MAE provisions and thirteen carve-outs included in our training sample. They range considerably in frequency across deals, from a high of $94.3 \%$ (for the affirmative MAC on business, operations, and financial condition - "MBOF") to less than 3\% (for affirmative MACs on target losses over a specified threshold - "MExessLos," and a MAC on the target's business prospects - "MPrspects"). Moreover, there appears to be considerable variation across categories, as indicated by the correlation matrix across provisions in Table 2, below. Most provisions have weak positive correlation with others, and the mean pair-wise correlation across terms is approximately 0.25 .

Our approach utilizes (and ultimately combines) two distinct methodological protocols for classifying textual data: (1) Regular expressions (RE) or "Boolean" protocols; and (2) Latent Semantic Analysis (LSA). We describe each of them below, ad seriatim the RE protocol we developed is based upon a hand-built "dictionary" of typical/characteristic grammatical patterns that frequently appear across texts, and which constitute alternative grammatical patterns to express a particular type of provision. Practically speaking, this dictionary very much has the look and feel of a compilation of search queries that are applied to the sample MAC/MAE documents for each coded term of interest. For example, in approximately $30 \%$ of the hand-coded data, the MAC/MAE contains a carve-out provision for "acts of God" (see Table 1). We found we were able to identify approximately three-quarters of these instances with a simple search protocol that tests for the proximity (e.g., within five words) of the word God and various conjugations of the word act. Other provisions, in contrast, exhibit greater linguistic and grammatical heterogeneity, and require more elaborate - and often more numerous - conditional search protocols. Thus, a substantial portion of our Python code consists of functions that invoke customized Boolean dictionaries to diagnose the presence or absence of each type

3 Unfortunately, Nixon Peobody does not keep track of individual coding attorneys, and we are therefore unable to control for any coder-specific biases. That said, we performed numerous hand audits of this data in order to ensure its consistency as much as possible (see discussion below). 
Table 1

MAC/MAE Terms and Relative Frequency in Data Set $(n=123$ Deals $)$

MAC/MAE Description

provision

\begin{tabular}{|c|c|c|}
\hline MBOF & $\begin{array}{l}\text { affirmative MAC on the business, operations, financial condition, } \\
\text { etc. }\end{array}$ & $94.3 \%$ \\
\hline MSelAbil & affirmative MAC on seller's ability to close the deal & $48.8 \%$ \\
\hline MExessLos & affirmative MAC for losses over a specified threshold & $2.4 \%$ \\
\hline MPrspects & affirmative MAC on prospects of the company/target & $2.4 \%$ \\
\hline MAssets & affirmative MAC on the securities or other assets of target & $21.1 \%$ \\
\hline MReasExp & $\begin{array}{l}\text { affirmative MAC triggered if there is reasonable expectation } \\
\text { of event to have a material adverse effect/change prospectively }\end{array}$ & $12.2 \%$ \\
\hline 1DispEffet & definition of materiality tied to "disproportionate effects" & $73.2 \%$ \\
\hline ChEcon & my or business $\mathrm{i}$ & $82.1 \%$ \\
\hline ChGen & specific industry & $79.7 \%$ \\
\hline ChSecM & exce & $63.4 \%$ \\
\hline EChPrVol & $\begin{array}{l}\text { change in trading price or trading volume of } \\
\text { tock }\end{array}$ & $52.0 \%$ \\
\hline EChI & r change in interests rates & $18.7 \%$ \\
\hline EChExch & hange rates & $14.6 \%$ \\
\hline EWar & major hostilities & $76.4 \%$ \\
\hline ETerror & exception for acts of terrorism & $79.7 \%$ \\
\hline EGod & exception for acts of God & $30.1 \%$ \\
\hline ERedCust & exception for reduction of & $29.3 \%$ \\
\hline EAnnTran & exception for effects of the announcement of the transaction & $71.5 \%$ \\
\hline EChAction & $\begin{array}{l}\text { exception for changes caused by the taking of any action required } \\
\text { or permitted or in any way resulting from or arising in connection } \\
\text { with the agreement }\end{array}$ & $70.7 \%$ \\
\hline EChGAAP & exception for changes in GAAP & $85.4^{c}$ \\
\hline
\end{tabular}

of lawyer-coded provision in each MAC/MAE. (As one might surmise, the process of building these dictionaries proved painstaking, involving nuanced reading of example provisions and common sense. We are still working on ways to further refine these dictionaries.) For notational housekeeping, consider provision $i$ in our data with lawyer-coded attributes $\left\{y_{i 1}, y_{i 2}, \ldots, y_{i k}\right\}$. Our regular expression protocol similarly generates a vector of binary diagnostic predictions about the presence of each sub-provisions of the MAC. We denote these predictions with $\left\{r_{i 1}, r_{i 2}, \ldots, r_{i k}\right\}$.

The second protocol was much less structured, and drew on machine-learning techniques from latent semantic analysis (LSA) literature. In contrast to RE, the LSA approach pays little heed to the grammatical architecture of a document, and concentrates instead on the "raw materials" (e.g., words) that compromise that document. To implement our LSA approach, our Python code extracted, for each MAC/MAE provision, a unigram frequency inventory - literally a "bag of words" 


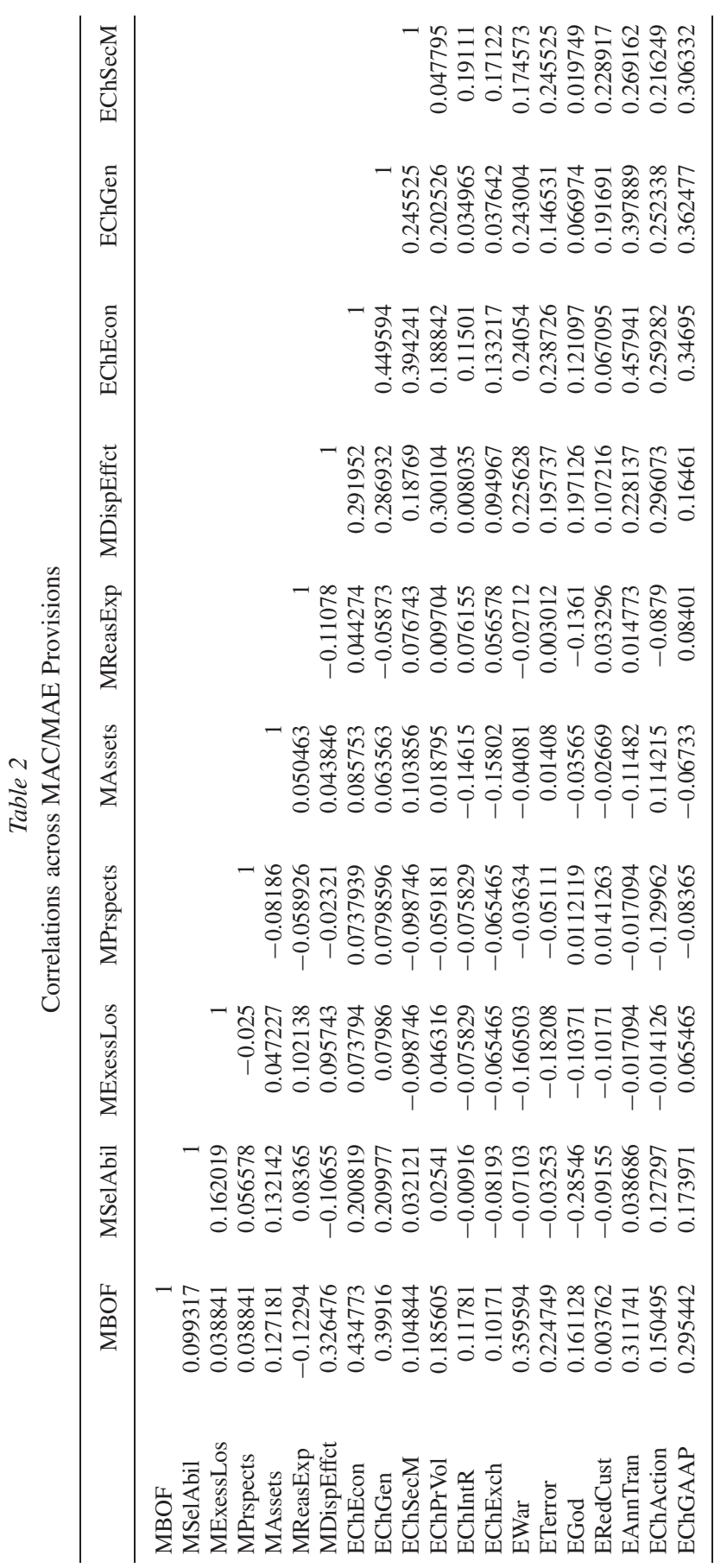




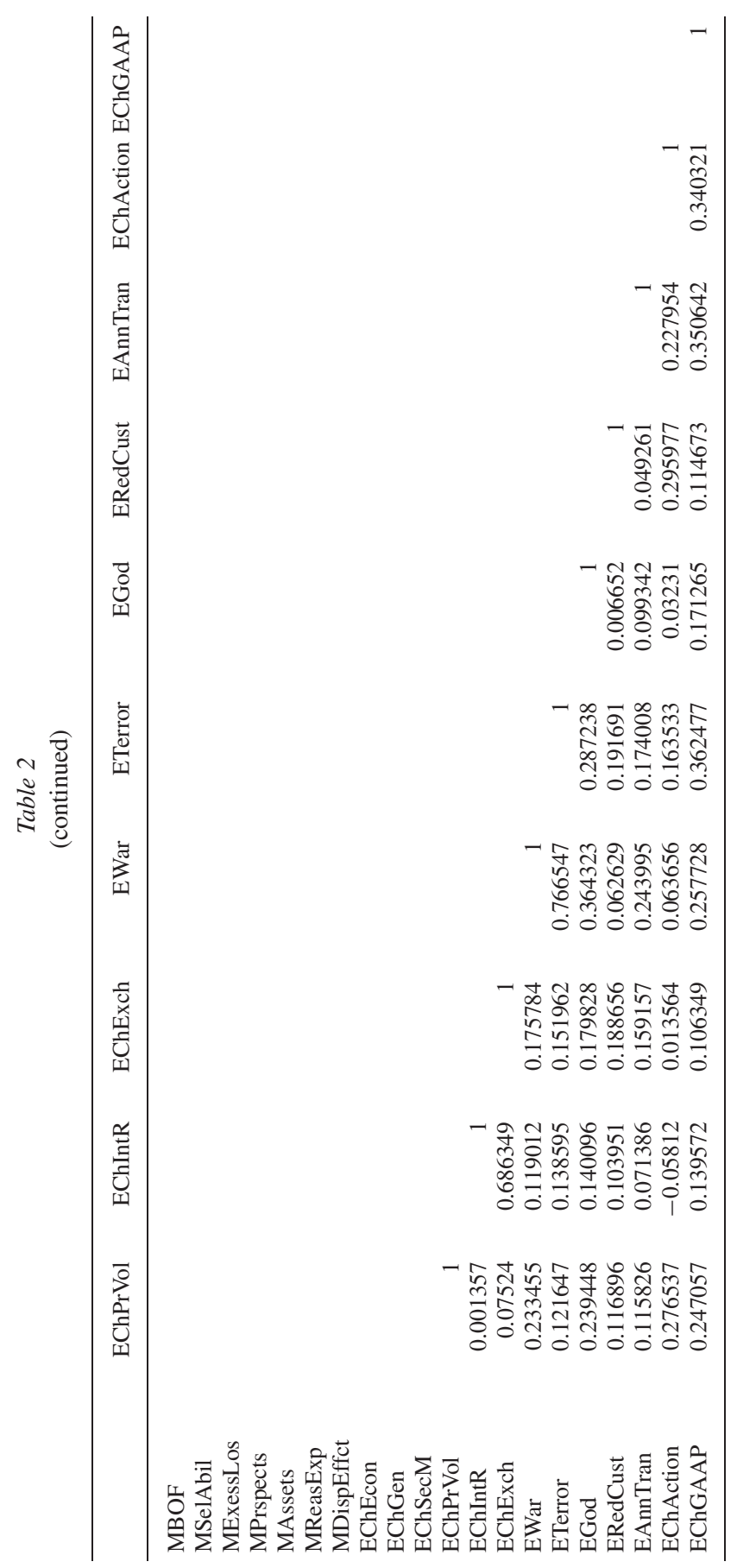


tabulating counts of each unique word across the entire set of deal documents. For the entire dataset, this process resulted in a term frequency matrix tracking the raw word counts of approximately 3,000 unique unigrams used across all documents. ${ }^{4}$ Denote this matrix by $\mathrm{N}$, where representative element $n_{i j}$ represents the number of times term $j$ appears in document $i$.

Next, we transformed the elements of $\mathrm{N}$ from their raw frequency counts into "term frequency - inverse document frequency" (or TF-IDF) measures. The resulting transformed matrix, T, contains representative element $t_{i j}$ for document $i$ and term $j$, defined by the expression

$$
t_{i j}=\left(\frac{n_{i j}}{\Sigma_{m} n_{m j}}\right) \times \ln \left[\frac{\left|\left\{j: n_{i j}>0\right\}\right|}{M}\right]^{-1},
$$

where $m \in\{1, \ldots, M\}$ indexes the universe of documents analyzed. The purpose and effect of this transformation is to accord greater proportional weight to terms that appear with large frequency in a particular document and yet are relatively uncommon overall. The first bracketed element of (1) represents the raw count of a given term in document $i$ relative to its total across all documents. The second term consists of the log of the inverse frequency with which term $j$ appears (at least once) across the universe (with cardinality $M$ ) of documents analyzed. By "rewarding" the frequent intra-document use of terms that are rare on the whole, the TF-IDF transformation tends to be better able to differentiate unique documents (Salton and Buckley, 1988). ${ }^{5}$

Because the TF-IDF transformation in (1) has a fixed point at $n_{i j}=0$, the transformed matrix $\mathrm{T}$ remains both extremely large and sparse. Following conventional approaches in LSA, we proceeded to reduce the dimensionality of T through singular value decomposition - a generalized form of principal component analysis. We retained the factors corresponding to the largest six eigenvalues from the decomposition. Ultimately, for each MAC/MAE provision $i$, our singular value decomposition of $\mathrm{T}$ resulted in the generation of factor matrix $\mathrm{X}$ with representative row $x_{i}$ consisting of the 6-tuple $\left\{x_{1 i}, x_{2 i}, \ldots, x_{6 i}\right\} .^{6}$

${ }^{4}$ In an unreported robustness check, we generated (and transformed) raw count not only for single-term unigrams, but also for bigrams and trigrams of consecutive terms. This alteration substantially increased computing time (generating over 30,000 word frequency variables), while only marginally enhancing the predictive power of our model. We therefore confine our analysis below to the case of single-term unigram frequencies.

5 We found little difference in results regardless of whether we utilized the matrix of raw unigram counts $\mathrm{N}$ or the TF-IDF transformed matrix T. Nevertheless, because this transformation is routinely applied in the natural language processing literature, we employ it in the analysis that follows.

6 Appendix A.2 provides a general description of the singular value decomposition approach. Note that the factors $x_{i}$ emerging from the singular value decomposition have no natural interpretive content, as they are merely algebraic artifacts of the underlying composition of T. Accordingly, we will spend no time exploring the intuition behind the estimated coefficients on $x_{i}$. 
We are now in a position to use the RE and/or LSA protocols to predict the presence of a specific term in the hand-coded database. As noted above, one interesting advantage of using both protocols is that it becomes possible to marshal their combined explanatory power in a hybrid predictive model. Thus, the baseline empirical specification for all of our predictive regressions is one that allows such combination, and is as follows. For each type $k$ of the twenty MAC/MAE provisions and exclusions described in Table 1, we estimate the following specification:

$$
\operatorname{Pr}\left\{y_{i k}=1\right\}=f\left(\alpha+\beta \cdot r_{i k}+\gamma \cdot x_{i}\right),
$$

where $y_{i k}, r_{i k}$, and $x_{i}$ are as described above, $f$ is a likelihood function, and $\alpha, \beta$, and $\gamma$ are estimated coefficients. All results reported below utilize a logistic specification for $f$ (though our results appear similar under probit and linear probability models as well).

\section{Tentative Results}

\subsection{Full-Sample Calibration}

Consider first the estimation of (2) across our entire dataset. This approach generates the most complete calibration of our machine-learning protocols to the training data set. (It does not, however, allow us for test for out-of-sample predictions, however - a question we turn to below.) Table 3 reports on the prediction characteristics of our logit estimation of equation (2). The table suppresses the direct logit estimation results, since the estimated coefficients are of little interest and most cannot be easily interpreted. Rather, it illustrates predictive performance across all 20 sampled MAC/MAE terms, and three different specifications of (2). (Thus, each row/panel entry in Table 3 represents a separate estimation.) In the first panel, we drop all the LSA variables, and regress the lawyer-coded attributes solely on our RE predictor. In the second panel, we drop the RE diagnostic and regress solely on the LSA factors. Finally, in the third panel, we include both the RE and the LSA factors in a "hybrid" model.

We consider two measures to evaluate predictive performance. First, we measure "correct" categorization rates using an assignment protocol that predicts the presence of a term in the training data if its predicted probability (at the estimated coefficients) exceeds a critical value of $1 / 2$. Second, we compute the Receiver Operating Characteristic (ROC) curve, and derive the area under the curve (AUC). (The ROC is a graphical plot of the false positive classification rate against the true positive classification rate as one continuously varies the critical probability threshold for prediction assignment from 0.0 to 1.0. A model that predicts nearly perfectly will exhibit a highly concave ROC curve, with an area under the curve of close to 1 . A model that makes nearly random predictions will exhibit a ROC that is approximately linear, with an area under the curve of close to 0.5 . ${ }^{7}$

\footnotetext{
7 As Hanczar et al. (2010) recently demonstrated, the ROC-AUC metric can suffer from being relatively noisy in smaller data sets. We nevertheless use it here as a gen-
} 
Table 3

Classification Rates and ROC-AUC for All Data

\begin{tabular}{|c|c|c|c|c|c|c|}
\hline \multirow[b]{2}{*}{$\begin{array}{l}\text { MAC/MAE } \\
\text { provision }\end{array}$} & \multicolumn{2}{|c|}{ RE/Boolean Specific. } & \multicolumn{2}{|c|}{ LSA Specification } & \multicolumn{2}{|c|}{ Hybrid Model } \\
\hline & $\begin{array}{l}\text { Correctly } \\
\text { classified }\end{array}$ & $\begin{array}{l}\text { ROC- } \\
\text { AUC }\end{array}$ & $\begin{array}{l}\text { Correctly } \\
\text { classified }\end{array}$ & $\begin{array}{l}\text { ROC- } \\
\text { AUC }\end{array}$ & $\begin{array}{l}\text { Correctly } \\
\text { classified }\end{array}$ & $\begin{array}{l}\text { ROC- } \\
\text { AUC }\end{array}$ \\
\hline $\mathrm{MBOF}$ & 0.9431 & 0.5505 & 0.9512 & {$[0.81]$} & 0.9512 & [0.8011] \\
\hline MSelAbil & 0.7886 & $\begin{array}{c}0.1034 \\
{[0.7865]} \\
0.0366\end{array}$ & 0.6260 & $\begin{array}{c}0.05 \\
{[0.6646]} \\
0.0492\end{array}$ & 0.7886 & $\begin{array}{c}0.0654 \\
{[0.8608]} \\
0.0336\end{array}$ \\
\hline MExessLos & 0.9754 & $\begin{array}{l}0.56 \\
0.132\end{array}$ & 0.9837 & $\begin{array}{c}{[0.8515]} \\
0.1029\end{array}$ & 0.9837 & $\begin{array}{c}{[0.8515]} \\
0.1029\end{array}$ \\
\hline MPrspects & 0.9756 & $\begin{array}{l}0.6625 \\
0.1667\end{array}$ & 0.9756 & $\begin{array}{c}{[0.8472]} \\
0.1132\end{array}$ & 0.9837 & $\begin{array}{c}{[0.8444]} \\
0.1288\end{array}$ \\
\hline MAssets & 0.7886 & $\begin{array}{c}{[0.6887]} \\
0.0489\end{array}$ & 0.7886 & $\begin{array}{l}0.5946 \\
0.0618\end{array}$ & 0.7967 & $\begin{array}{c}{[0.7179]} \\
0.0552\end{array}$ \\
\hline MReasExp & 0.8760 & $\begin{array}{c}0.51 \\
0.1911\end{array}$ & 0.8943 & $\begin{array}{l}0.6596 \\
0.0815\end{array}$ & 0.9008 & $\begin{array}{c}{[0.6664]} \\
0.0805\end{array}$ \\
\hline MDispEffct & 0.7317 & $\begin{array}{l}0.5965 \\
0.0506\end{array}$ & 0.7561 & $\begin{array}{c}{[0.7215]} \\
0.0593\end{array}$ & 0.7886 & $\begin{array}{c}{[0.7182]} \\
0.0603\end{array}$ \\
\hline EChEcon & 0.8211 & $\begin{array}{l}0.5511 \\
0.0584\end{array}$ & 0.8537 & $\begin{array}{c}{[0.7052]} \\
0.0614\end{array}$ & 0.8537 & $\begin{array}{c}{[0.7066]} \\
0.0613\end{array}$ \\
\hline EChGen & 0.7967 & $\begin{array}{c}0.572 \\
0.0557\end{array}$ & 0.8049 & $\begin{array}{c}{[0.6865]} \\
0.0619\end{array}$ & 0.8211 & $\begin{array}{c}{[0.6918]} \\
0.0607\end{array}$ \\
\hline EChSecM & 0.7317 & $\begin{array}{c}{[0.7462]} \\
0.04\end{array}$ & 0.6260 & $\begin{array}{l}0.5942 \\
0.0526\end{array}$ & 0.7642 & $\begin{array}{c}{[0.8132]} \\
0.0407\end{array}$ \\
\hline EChPrVol & 0.7398 & $\begin{array}{c}{[0.7427]} \\
0.0391\end{array}$ & 0.6829 & $\begin{array}{c}{[0.7299]} \\
0.0454\end{array}$ & 0.7724 & $\begin{array}{c}{[0.8292]} \\
0.0381\end{array}$ \\
\hline EChIntR & 0.8618 & $\begin{array}{c}{[0.6974]} \\
0.0538\end{array}$ & 0.8211 & $\begin{array}{c}{[0.7633]} \\
0.0499\end{array}$ & 0.8618 & $\begin{array}{c}{[0.8376]} \\
0.0455\end{array}$ \\
\hline EChExch & 0.9024 & $\begin{array}{c}{[0.7817]} \\
0.06\end{array}$ & 0.8699 & $\begin{array}{c}{[0.8153]} \\
0.0556\end{array}$ & 0.9106 & $\begin{array}{l}{[0.882]} \\
0.0562\end{array}$ \\
\hline EWar & 0.7642 & $\begin{array}{c}{[0.6812]} \\
0.049\end{array}$ & 0.7724 & $\begin{array}{c}{[0.7196]} \\
0.0464\end{array}$ & 0.7642 & $\begin{array}{c}{[0.7929]} \\
0.0432\end{array}$ \\
\hline ETerror & 0.7967 & $\begin{array}{c}{[0.7461]} \\
0.0414\end{array}$ & 0.8130 & $\begin{array}{c}{[0.7276]} \\
0.0513\end{array}$ & 0.8049 & $\begin{array}{c}{[0.8137]} \\
0.0435\end{array}$ \\
\hline EGod & 0.7724 & $\begin{array}{c}{[0.714]} \\
0.0454\end{array}$ & 0.7317 & $\begin{array}{c}{[0.7329]} \\
0.0494\end{array}$ & 0.7724 & $\begin{array}{c}{[0.7986]} \\
0.0462\end{array}$ \\
\hline ERedCust & 0.8862 & $\begin{array}{c}{[0.8788]} \\
0.0355\end{array}$ & 0.7236 & $\begin{array}{c}{[0.7701]} \\
0.0474\end{array}$ & 0.8943 & $\begin{array}{c}{[0.9132]} \\
0.0319\end{array}$ \\
\hline EAnnTran & 0.7154 & $\begin{array}{c}{[0.6153]} \\
0.0495\end{array}$ & 0.7236 & {$[0.6722]$} & 0.7317 & [0.6946] \\
\hline EChAction & 0.7073 & $\begin{array}{c}{[0.6652]} \\
0.0478\end{array}$ & 0.7073 & $\begin{array}{l}{[0.095]} \\
0.0584\end{array}$ & 0.7073 & $\begin{array}{c}{[0.7663]} \\
0.0475\end{array}$ \\
\hline
\end{tabular}

Notes: Baseline Regression is as in equation (2), which is estimated for each provision against three specifications: (a) RE Predictors only, (b) LSA Factors only, and (c) hybrid RE and LSA. The classification protocol in the first column of each panel assigns term as present if the computed marginal probability evaluated at the estimated coefficients exceeds 0.5 . The second column of each specification reports estimates of the area under the ROC. Standard errors are beneath, in italics. Coefficients that are statistically significant at the 0.05 level are in square brackets. 
Our estimates produce a respectable (though still imperfect) rate of correct classification in replicating the Nixon Peabody data set across the 123 sampled deals. Overall, each of the RE and LSA approaches were able to match the sample terms with an average $80 \%$ accuracy rate. On a term-by-term basis, our mismatch rate range between zero and thirty-seven percent. (Not surprisingly, misclassifications in the RE approach are skewed towards false negatives, while they are generally balanced between false negatives and false positives in the LSA estimations.) On the whole, the LSA estimates tended to yield slightly larger correct classification rates than the RE estimates, though in a few situations the LSA predictors preformed much worse. When the RE and LSA approaches were combined, however, classification accuracy generally increased (to $84 \%$ across categories), and in some cases the improvement was dramatic.

The ROC-AUC measures suggest a similar pattern. In all our specifications, estimated ROC areas indicated that each of our approaches is diagnostically probative. Moreover, the combination of RE and LSA approaches delivers a discernible increase in predictive performance across nearly all coded terms. We view these base results as a promising start, but one that can be significantly improved upon with more consultative and programming attention. In fact, there may be a sense in which these figures understate the accuracy of our approach, since the Nixon Peabody data invariably will contain undetected coding errors. Auditing some of the evident mismatches, we discovered that Nixon Peabody attorneys appear to have had a mis-coding rate in excess of 3\%. Consequently, it is plausible that Table 3 understates the correct categorization rate relative to the "true" underlying contractual terms. $^{8}$

\subsection{Out-of-Sample Monte Carlo Simulations}

Although Table 3 illustrates the explanatory power gained by marshaling both regular expression and latent semantic techniques for predicting the presence/absence of particular terms, its results are distinctly within sample. They need not (and likely do not) remain as strong when the predictive model is taken outside of the sample constellation of deals. Yet it is predominantly in out-of-sample prediction where our approach can be useful in economizing the time and expense of hand coding. The discussion below, therefore, considers out-of-sample prediction issues more squarely.

In order to simulate out-of-sample prediction, we employed a Monte Carlo bootstrap aggregation ("bagging”) approach proposed by Breiman (1996); Friedman, Hastie, and Tibshirani $(2000,2003)$. We devised an identical bagging protocol for

eral guidepost, especially in light of the absence of alternative good measures of predictive probity.

8 This assertion, of course, requires significantly more investigation. For example, we have not yet audited any of the evidently correctly matched terms to determine whether both the Nixon Peabody data as well as our own protocol are mis-coding some deals. 
Table 4

Monte Carlo Simulation for Out-of-Sample Prediction

\begin{tabular}{|c|c|c|c|c|}
\hline \multirow[b]{2}{*}{ MAC/MAE provision } & \multicolumn{2}{|c|}{ LSA Specification } & \multicolumn{2}{|c|}{ Hybrid Model } \\
\hline & Corr. class & ROC-AUC & Corr. class & ROC-AUC \\
\hline \multirow[t]{2}{*}{ MBOF } & [0.913] & 0.61587 & [0.9284] & 0.5327 \\
\hline & 0.04744501 & 0.2975 & 0.04117 & 0.27813 \\
\hline \multirow[t]{2}{*}{ MSelAbil } & 0.54222 & 0.5599 & [0.7421667] & {$[0.783655]$} \\
\hline & 0.08408 & 0.0882 & 0.070876 & 0.0784866 \\
\hline \multirow[t]{2}{*}{ MAssets } & {$[0.764333]$} & 0.6345 & {$[0.764]$} & 0.58404 \\
\hline & 0.0675466 & 0.1017 & 0.068276 & 0.10996 \\
\hline \multirow[t]{2}{*}{ MReasExp } & {$[0.864033]$} & 0.46393 & [0.8777333] & 0.49465 \\
\hline & 0.058078 & 0.16247 & 0.0555032 & 0.190937 \\
\hline \multirow[t]{2}{*}{ MDispEffct } & {$[0.7316]$} & 0.6609 & [0.7357333] & 0.6320073 \\
\hline & 0.067958 & 0.10718 & 0.07652223 & 0.1193881 \\
\hline \multirow[t]{2}{*}{ EChEcon } & {$[0.8209]$} & 0.5367427 & [0.8201667] & 0.6198363 \\
\hline & 0.05871753 & 0.1302458 & 0.06222236 & 0.131313 \\
\hline \multirow[t]{2}{*}{ EChGen } & [0.7823333] & 0.5047211 & [0.7692] & 0.583982 \\
\hline & 0.06921313 & 0.1315335 & 0.06542236 & 0.1247254 \\
\hline \multirow[t]{2}{*}{ EChSecM } & 0.5816 & 0.4832542 & [0.7038667] & [0.7294512] \\
\hline & 0.07611767 & 0.09772 & 0.07128283 & 0.0914256 \\
\hline \multirow[t]{2}{*}{ EChPrVol } & 0.5678333 & 0.6334498 & {$[0.7281]$} & {$[0.7751]$} \\
\hline & 0.07982975 & 0.087661 & 0.07246226 & 0.0852973 \\
\hline \multirow[t]{2}{*}{ EChIntR } & [0.7874667] & 0.6621522 & {$[0.8251]$} & 0.7240246 \\
\hline & 0.06722013 & 0.132249 & 0.06456472 & 0.1269156 \\
\hline \multirow{2}{*}{ EChExch } & [0.8140667] & 0.5999294 & {$[0.8785]$} & {$[0.805348]$} \\
\hline & 0.0616201 & 0.1569705 & 0.05493118 & 0.1496069 \\
\hline \multirow{2}{*}{ EWar } & [0.7290333] & 0.5679435 & {$[0.7242]$} & {$[0.7217855]$} \\
\hline & 0.06955984 & 0.100249 & 0.07423599 & 0.0949628 \\
\hline \multirow[t]{2}{*}{ ETerror } & [0.7728333] & 0.5361141 & [0.7530333] & {$[0.7372462]$} \\
\hline & 0.06653955 & 0.1150063 & 0.07099496 & 0.1141708 \\
\hline \multirow[t]{2}{*}{ EGod } & [0.6942333] & 0.5840398 & [0.7248333] & {$[0.727878]$} \\
\hline & 0.07621825 & 0.1060234 & 0.07321809 & 0.097808 \\
\hline \multirow[t]{2}{*}{ ERedCust } & [0.7230333] & [0.7399407] & [0.8596333] & [0.8586069] \\
\hline & 0.08007837 & 0.0928146 & 0.05638499 & 0.0849821 \\
\hline \multirow[t]{2}{*}{ EAnnTran } & [0.6908667] & 0.3879988 & [0.6821667] & 0.6091421 \\
\hline & 0.07399589 & 0.1039311 & 0.07364824 & 0.110092 \\
\hline \multirow[t]{2}{*}{ EChAction } & [0.7089] & 0.6196205 & {$[0.6671]$} & [0.6711904] \\
\hline & 0.0730488 & 0.105946 & 0.07533583 & 0.1100768 \\
\hline
\end{tabular}

Notes: Baseline regression is as in equation (2), estimated 1000 times on a "training" dataset containing a 75\% sample (sampled randomly, for each iteration), and generating (simulated) out-of-sample predictions for the remaining 25\%. For each term, the simulation explores two specifications: (a) LSA factors only; and (b) hybrid RE and LSA. The classification protocol in the first column of each panel assigns term as present if the computed marginal probability evaluated at the estimated coefficients exceeds 0.5 . The second column of each specification reports estimates of the area under the ROC. Empirical standard errors appear beneath, in italics. (The MAC terms MExessLos and MPrspects could not be simulated reliably because of their low-frequency representation in the data). Coefficients that are statistically significant at the 0.05 level are in square brackets. 
each contractual provision studied (i.e., each of the 20 affirmative MACs/MAEs and exceptions). Within each Monte Carlo iteration, the data were randomly segregated into two groups: A provisional "training" dataset, consisting of roughly $75 \%$ of our observations, and a provisional "testing" dataset, consisting of the remaining $25 \%$ of the data. We then fit equation (2) to the training data using (successively) LSA and hybrid approaches. And as before, we used the resulting coefficient estimates to generate probabilistic predictions of the presence/absence of the contractual term at issue in the remaining testing data, generating estimates of both correct classification rates, and of ROC-AUCs. For each of the 20 terms studied, we repeated the Monte Carlo simulation 1,000 times. ${ }^{9}$ (Each succeeding iteration re-sampled our training/testing data with replacement.) Table 4 reports on the resulting empirical distributions of both classification rates and ROC-AUCs. (Note that the table reports only on the "pure" LSA and the hybrid model, excluding the pure RE model - given the way that the RE dictionary was assembled, Monte Carlo methods were not informative for the pure RE model).

As expected, the out-of-sample predictions in Table 4 are weaker and noisier than the full-sample calibrations of Table 4. Moreover, for two provisions (MExessLoss and MPrspects), there was simply not enough data variability within the sample to execute the Monte Carlo estimations consistently. Nevertheless, for the remaining 18 terms, our simulated out-of-sample predictions appear to remain relatively strong. Average correct classification rates across all terms are approximately $73.5 \%$ for the LSA specification and $78 \%$ for the hybrid specification. The ROC-AUC measures also appear relatively good - only slightly smaller numerically than those in Table 3, but subject to considerably more noise.

In unreported robustness checks, we reran the bagging protocol with varying proportions of training/testing data. The results were largely consistent - though they tended to weaken as our training data sampled smaller proportions of the entire sample. We conjecture that as we add additional MAC/MAE provisions to our database, we will be able to enhance this performance significantly.

\section{Discussion, Extensions, and Conclusion}

Although we consider the above exercise to be a successful proof of concept, it is limited by a number of factors - not the least of which is sample size. With only 123 coded deals to work with, we were unable to marshal much of the potential power of both RE and LSA protocols (particularly the latter) in calibrating our predictive models. We are currently working to expand the size of our pilot data over time and cross-sectionally.

Similarly, we have limited our attention here to deals that are both publicly available and coded in the Nixon Peabody dataset. An obvious follow-on step would be to take our predictive model outside this data set, applying it to all

\footnotetext{
9 Based on preliminary investigations, very little additional accuracy emerged from increasing the number of iterations to either 10,000 or 100,000 .
} 
publicly available merger agreements (be they included in the training data or not). We are currently in the process of implementing this step for the years 2007-10, and will report on results in subsequent work.

A third extension of our approach would be to utilize alternative existing data to provide an additional calibration device for tokenizing a larger database. Although the Nixon Peabody data is rich, detailed, and coded by practicing lawyers, it is not unique. In particular the American Bar Association also harvests a regular survey of M\&A agreements (including MAC/MAE clauses) that could provide either a useful calibration check, or additional coding attributes not available in the Nixon Peabody data.

Yet another factor that constrains our analysis concerns the inherent limitations in using a training data set (Nixon Peabody, ABA, or something else) that is not collected under conventional experimental conditions. As noted above, we were unable to exercise control over either the conditions under which lawyers coded this data, or the targets of their efforts. Thus, we were unable to track subjectspecific effects or other systemic factors that may have affected the reliability of the coding efforts. Moreover, we could not glean other - potentially more interesting - metrics for the breadth and content of a MAC/MAE provision. For instance, the Nixon Peabody data does not elicit an all-things-considered assessment of whether a particular provision is buyer- or seller-friendly, or how complex and unpredictable its application might be in practice. We are currently piloting a more controlled experimental instrument that attempts to elicit this information, and which will constitute a separate paper from this one.

A related issue concerns the incidence of human coding errors in the Nixon Peabody data set (notwithstanding its established expertise in the field). As previously noted, our machine-learning protocol is calibrated against this lawyer-coded data, and accordingly coding errors in the Nixon Peabody data can easily propagate similar errors in our own model. Notwithstanding this real possibility, it is also important to point out that our approach may also represent an important resource for improving the accuracy of the hand-coded data. Indeed, our predictive model allowed us to audit poorly predicted provisions by hand to determine the source of the inconsistency. This process allowed us to discover a small number $(\sim 3 \%)$ of systematic errors in Nixon Peabody's coding data. In this way, while our project has attempted to duplicate Nixon Peabody's results, we have also attempted to improve the underlying coding algorithms created by Nixon Peabody. Our approach also represents a means for rapidly prototyping and testing coding algorithms that can assist human coders in drawing out syntactical nuance in legal language. The ability to prototype and test a candidate syntactical form could also speed the creation of hand-coded documents, and perhaps lead to a deeper understanding of M\&A agreement architecture.

Notwithstanding its significant recent growth, data-driven empirical methods in legal scholarship have only begun to scratch the surface of their ultimate capabilities. Litigated cases, appellate opinions, and Supreme Court decisions - the chief targets of much of the current ELS literature - are but a tiny fraction of what attorneys 
actually do in practice. Much transactional work, particularly within business law, is only now beginning to lend itself to serious quantitative analysis. This project presents some initial steps in pushing those efforts forward in the M\&A context, and in a way that facilitates the harvesting of data at a lower cost, with greater consistency, and more dynamic adaptability than is currently the available. Based on our results thus far, we are optimistic that we can implement our protocol on a wide scale basis, not only to understand the nature and evolution of MAC/MAE clauses (an important topic itself), but also to facilitate the harvesting of data across a large array of legal transactional domains.

\section{Appendix}

\section{A.1 Screen Shot from Pilot Study: Python-Based Interface}

This screen shot displays a typical user interface as the program scours a sample acquisition agreement. In this screen shot, the program detects three species of MAC/MAE provisions, and fails to find seven others.

Figure

Screen Shot of Text Analysis Platform

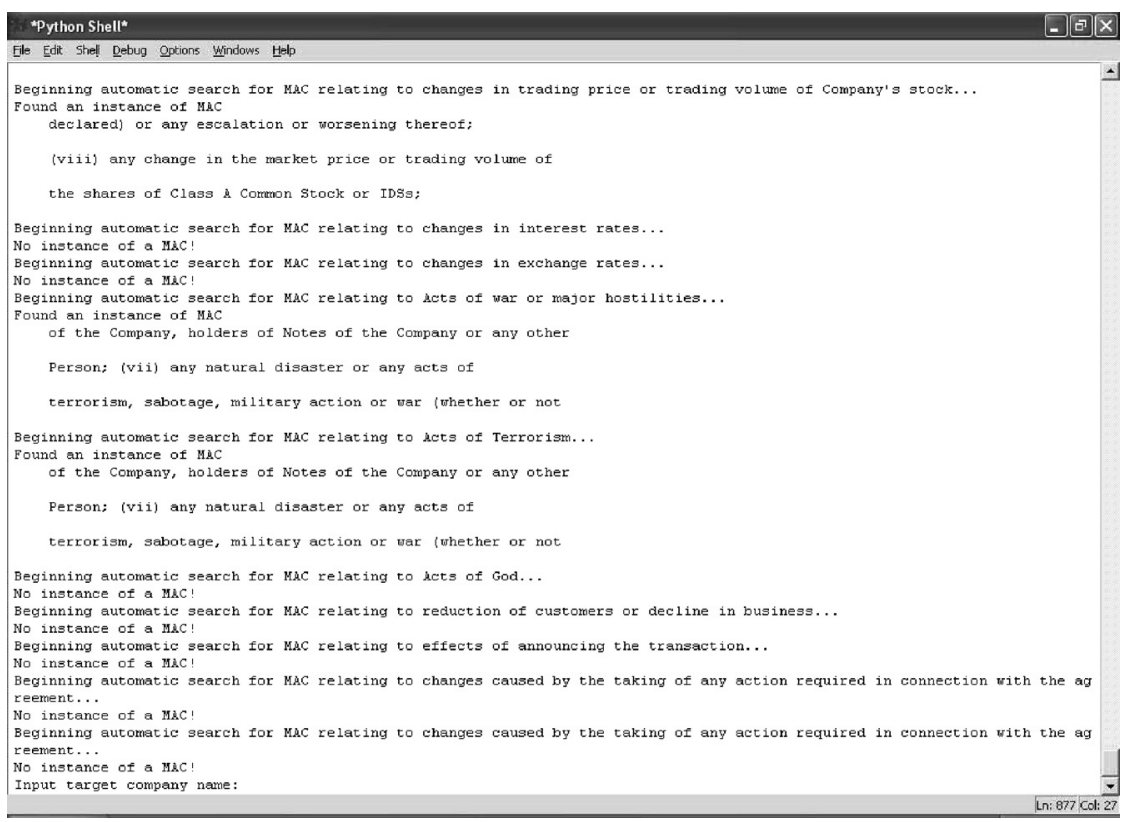




\section{A.2 Singular Value Decomposition Description}

The LSA approach in the text required us to analyze a unigram matrix of TF-IDF transformed word counts for all deals in our data set. While the number of deals is modest, at only 123 documents, the word frequency data alone that was gathered from a single document consisted of $\sim 3000$ recorded frequencies. A common technique used to simplify analytics in such large is to reduce the dimensionality of the data set by using singular value decomposition (a generalization of principal component analysis). The concept is fairly simple. Consider our transformed TF-IDF word count matrix, denoted as T (with elements $t_{i j}$ ). Singular value decomposition (or SVD) involves using the algebraic structure of $\mathrm{T}$ to produce synthetic variables that are designed to explain internal variation within $\mathrm{T}$. The key algebraic relationship for accomplishing this task is given by the decomposition:

$$
\mathrm{T}=\mathrm{U}^{*} \mathrm{~S}^{*} \mathrm{X}^{\mathrm{T}},
$$

where $\mathrm{U}$ is an orthonormal basis which spans the rows of the original matrix $\mathrm{T}, \mathrm{V}$ is an orthonormal basis which spans the columns of $\mathrm{T}$, and $\mathrm{S}$ is the matrix of eigenvalues.

Each succeeding row of $\mathrm{X}^{\mathrm{T}}$ (column of $\mathrm{X}$ ) corresponds to a synthesized variable capturing successively smaller sources of internal data variation. LSA typically chooses the first $k$ components (here, we retain $k=6$ components). Beyond this description, we do not have sufficient space to lay out the mathematical basis of SVD, but instead refer the reader to some concepts from linear algebra. See, e.g., Bishop (2006).

\section{References}

Bishop, C. M. (2006), Pattern Recognition and Machine Learning, Springer Science + Business Media LLC, New York.

Breiman, L. (1996), "Bagging Predictors," Machine Learning, 24, 123-140.

Choi, A., and G. Triantis (2010), "Strategic Vagueness in Contract Design: The Case of Corporate Acquisitions" Yale Law Journal, 119, 848-924.

Friedman, J., T. Hastie, and R. Tibshirani (2000), "Additive Logistic Regression: A Statistical View of Boosting," The Annals of Statistics, 28(2), 337-407.

_, - , and - (2003), The Elements of Statistical Learning: Data Mining, Inference, and Prediction, Springer Series in Statistics, Springer Science + Business Media LLC, New York.

Gilson, R., and A. Schwartz (2005), "Understanding MACs: Moral Hazard in Acquisitions," The Journal of Law, Economics, \& Organization, 21(2), 330-358.

Hanczar, B., J. Hua, C. Sima, J. Weinstein, M. Bittner, and E. R. Dougherty (2010), "SmallSample Precision of ROC-Related Estimates," Bioinformatics, 26(6), 822-830.

Macias, A. J. (2009), "Risk Allocation and Flexibility in Acquisitions: The Economic Impact of Material-Adverse-Change (MACs) Clauses," AFA 2009 San Francisco Meetings Paper, available at http://ssrn.com/abstract $=1108792$.

Nixon Peabody LLP (2008), "Seventh Annual MAC Survey," available at http://www. nixonpeabody.com/publications_detail3.asp?ID=2488.

Salton, G., and C. Buckley (1988), "Term-Weighting Approaches in Automatic Text Retrieval," Information Processing \& Management, 24(5), 513-523. 
Talley, E. (2009), "On Uncertainty, Ambiguity, and Contractual Conditions," The Delaware Journal of Corporate Law, 34(3), 755-812.

Eric Talley

Drew O'Kane

School of Law

University of California, Berkeley

Berkeley, CA 94705

U.S.A.

E-mail:

etalley@law.berkeley.edu

deokane@gmail.com 
